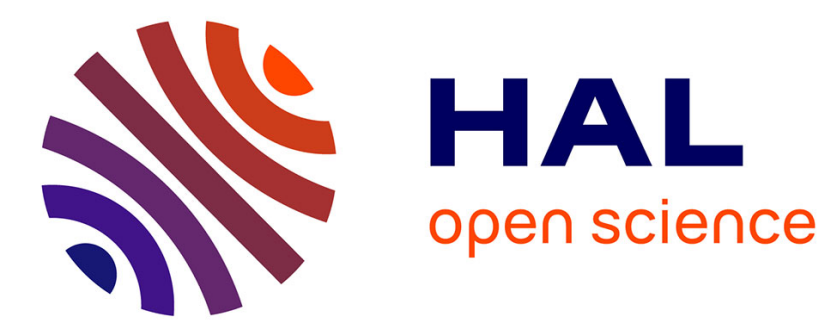

\title{
Spin polarization as an electronic cooperative effect
}

Nadia Ben Amor, Camille Noûs, Georges Trinquier, Jean-Paul Malrieu

\section{To cite this version:}

Nadia Ben Amor, Camille Noûs, Georges Trinquier, Jean-Paul Malrieu. Spin polarization as an electronic cooperative effect. Journal of Chemical Physics, 2020, 153 (4), pp.044118. 10.1063/5.0011582 . hal-02958773

\section{HAL Id: hal-02958773 \\ https://hal.science/hal-02958773}

Submitted on 6 Oct 2020

HAL is a multi-disciplinary open access archive for the deposit and dissemination of scientific research documents, whether they are published or not. The documents may come from teaching and research institutions in France or abroad, or from public or private research centers.
L'archive ouverte pluridisciplinaire HAL, est destinée au dépôt et à la diffusion de documents scientifiques de niveau recherche, publiés ou non, émanant des établissements d'enseignement et de recherche français ou étrangers, des laboratoires publics ou privés. 


\section{Spin polarization as an electronic cooperative effect}

Cite as: J. Chem. Phys. 153, 044118 (2020); https://doi.org/10.1063/5.0011582

Submitted: 22 April 2020 . Accepted: 22 June 2020 . Published Online: 28 July 2020

Nadia Ben Amor (D), Camille Noûs, Georges Trinquier (D), and Jean-Paul Malrieu (D)

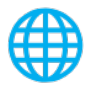

\section{ARTICLES YOU MAY BE INTERESTED IN}

The density matrix renormalization group in chemistry and molecular physics: Recent developments and new challenges

The Journal of Chemical Physics 152, 040903 (2020); https://doi.org/10.1063/1.5129672

Spin chemistry

The Journal of Chemical Physics 152, 120401 (2020); https://doi.org/10.1063/5.0006547

Hierarchical machine learning of potential energy surfaces

The Journal of Chemical Physics 152, 204110 (2020); https://doi.org/10.1063/5.0006498
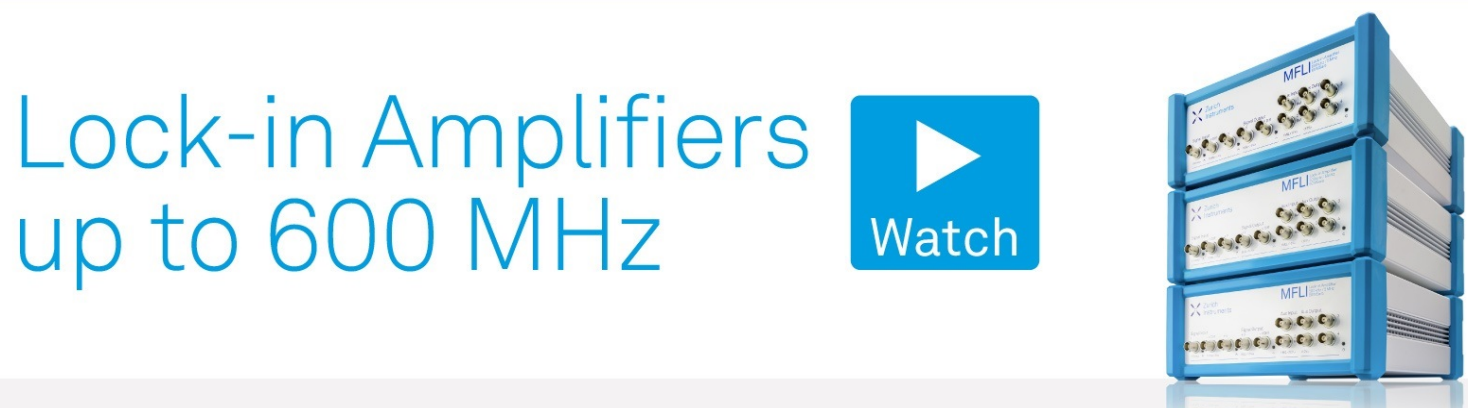

J. Chem. Phys. 153, 044118 (2020); https://doi.org/10.1063/5.0011582 


\title{
Spin polarization as an electronic cooperative effect
}

\author{
Cite as: J. Chem. Phys. 153, 044118 (2020); doi: 10.1063/5.0011582 \\ Submitted: 22 April 2020 - Accepted: 22 June 2020 • \\ Published Online: 28 July 2020
}

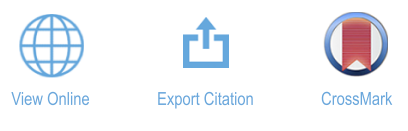

Nadia Ben Amor, ${ }^{1, a)}$ (D) Camille Noûs, ${ }^{2}$ Georges Trinquier, ${ }^{1}$ (D) and Jean-Paul Malrieu' (D)

\author{
AFFILIATIONS \\ ${ }^{1}$ Laboratoire de Chimie et Physique Quantiques, IRSAMC-CNRS-UMR5626, Université Paul-Sabatier (Toulouse III), \\ 31062 Toulouse Cedex 4, France \\ ${ }^{2}$ Laboratoire Cogitamus IRSAMC-CNRS-UMR5626, Université Paul-Sabatier (Toulouse III), 31062 Toulouse Cedex 4, France
}

a) Author to whom correspondence should be addressed: benamor@irsamc.ups-tlse.fr

\begin{abstract}
Taking as an example the simple $\mathrm{CH}_{3}$ radical, this work demonstrates the cooperative character of the spin-polarization phenomenon of the closed-shell core in free radicals. Spin polarization of $\mathrm{CH} \sigma$ bonds is not additive here, as spin polarization of one bond enhances that of the next bond. This cooperativity is demonstrated by a series of configuration interaction calculations converging to the full valence limit and is rationalized by analytic developments. The same phenomenon is shown to take place in those diradicals where spin polarization plays a major role, as illustrated in square planar carbo-cyclobutadiene $\mathrm{C}_{12} \mathrm{H}_{4}$. The treatment of cooperativity represents a challenge for usual post-Hatree-Fock methods.
\end{abstract}

Published under license by AIP Publishing. https://doi.org/10.1063/5.0011582

\section{INTRODUCTION}

This work is devoted to the theoretical treatment of the spinpolarization phenomenon. It first recalls its manifestation in free radicals and, in particular, in conjugated hydrocarbons, where ESR experiments could only be interpreted by considering this effect. ${ }^{1-}$ Taking the paradigmatic example of a $\mathrm{CH}_{3}$ radical, the occupation numbers of the exact wave functions definitely support the basic description of these monoradicals as the product of a closed-shell core [doubly occupied molecular orbitals (MOs)] and a singly occupied MO (SOMO), the corresponding electron having a well-defined $m_{s}$ value, being, for instance, of spin-up character. Starting from this single-reference determinant, the spin polarization is brought by the single excitations that introduce the differential effect of the exchange integrals between the core electrons and the unpaired electron, depending on the spin of the core electrons. Even when one involves spin-flip determinants, which change the spin of the unpaired electron and excite the core to a triplet state, the phenomenon is a priori confined to the so-called one-hole-one-particle (1h-1p) excitations. These points are addressed in Sec. II. From a series of configuration interaction (CI) calculations involving higher and higher excitation levels, we show that the coefficients of $1 \mathrm{~h}-1 \mathrm{p}$ determinants systematically increase when one goes to higher excitation degrees. These are unexpected and counterintuitive results since $a$ priori the wave function is diluted in a larger number of determinants, and this is, indeed, in contrast with a systematic decrease in the coefficient of the reference function. This proves that higher excitations increase the weight of the single excitations. Section II tries to interpret this behavior, apparently resulting from two mechanisms. The first one is an interplay between correlation and spin polarization, some double excitations increasing the coefficients of spin-polarizing determinants. The other contribution may be seen as a cooperative effect since spin polarization of a given shell is enhanced by that of another shell. This is the manifestation of a cooperative effect-a rare phenomenon in electronic populations of molecules.

In Sec. III, the same analysis is applied to diradicals, where two orbitals are singly occupied, generating singlet and triplet states. Depending on their spin-organization, the two unpaired electrons induce a differential exchange field on the core electrons, and the spin-polarization of the core is an important component of the singlet-triplet gap. A dramatic example is the case of the $90^{\circ}$ twisted ethylene molecule, where the singlet is below the triplet state. ${ }^{5,6}$ While in the triplet state, the spin polarization may be considered as "static," at least in its $m_{s}= \pm 1$ components, that of the singlet is sometimes called "dynamic," but this difference is 
artificial since it has the same status in the $m_{s}=0$ component of the triplet.

The cooperative character of the spin polarization is scrutinized on a diradical situation exemplified by the conjugated system carbo-cyclobutadiene $\mathrm{C}_{12} \mathrm{H}_{4}(-\mathrm{CH}-\mathrm{C} \equiv \mathrm{C}-)_{4}$ in $D_{4 h}$ symmetry. The energy of its singlet state is dramatically stabilized by spin polarization of the doubly occupied $\pi$ MOs, and a series of configuration interaction (CI) calculations involving higher and higher excitation levels again show that the coefficients of $1 \mathrm{~h}-1 \mathrm{p}$ determinants systematically increase when one goes to higher excitation degrees up to the full valence $\mathrm{CI}$.

Finally, in Sec. IV, we will show why such phenomena represent a challenge to the famous (and, in general, firmly grounded) coupled cluster approaches, which rest on the additivity of excitation energies and transferability of the interactions of the same operator applied on determinants of various excitation levels. The specificity of this cooperative effect will be discussed, which is "interaction driven," in contrast to other cooperative effects that are "excitation-energy" driven.

\section{SPIN POLARIZATION EFFECT IN MONO-RADICALS}

The standard description of mono-radicals starts from a single determinant where the unpaired electron occupies a SOMO $a$, while the other electrons are paired in a closed-shell core,

$$
\Phi_{R}=\left|a \Pi_{k} k \bar{k}\right| .
$$

In this restricted single-determinant wave function, the spin density is simply expressed from the SOMO, $\rho(r)=a(r)^{2}$.

The Fock operator for the closed-shell MOs is given by

$$
F=h+J_{a}-K_{a} / 2+\sum_{k}\left(2 J_{k}-K_{k}\right) .
$$

The concept of spin-polarization was introduced to rationalize the properties of the simplest radical hydrocarbon, namely, $\mathrm{CH}_{3}{ }^{\circ}$. In this radical, which locates the four nuclei in a plane of symmetry, say, the $x y$ plane, the SOMO reduces to a $2 \mathrm{p}_{z}$ type orbital on the carbon atom, as pictured in Fig. 1. It has zero amplitude in the plane and thus on the $\mathrm{H}$ protons. However, electron paramagnetic resonance experiments detect an interaction between the electron spin and proton spins, meaning that the spin density is non-zero in the $x y$ plane. McConnell et al. and other authors simultaneously rationalized this phenomenon and named it "spin-polarization of the doubly occupied MOs." ${ }^{1-4}$ This problem suggested the relevance of unrestricted or broken symmetry (BS) self-consistent descriptions. ${ }^{7-10}$

\section{A. First-order approach}

The occurrence of a spin density in the plane of symmetry may be rationalized along two directions. The most general and exact one consists in a CI expansion of the wave function. A simpler one consists in leaving the frozen character of the core, optimizing the energy of a single determinant in which the $\alpha$ and $\beta$ spin electrons of the core have different space parts. This determinant no longer respects the spin symmetry (namely, the character of eigenfunction of the $S^{2}$ operator) and is designated as broken-symmetry (or unrestricted),

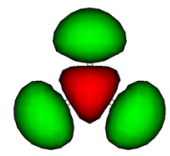

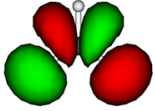

5
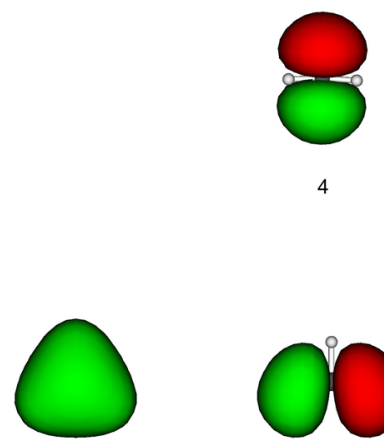

1

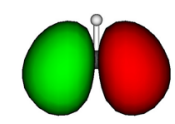

2


3
FIG. 1. Molecular orbitals of the $\mathrm{CH}_{3}$ radical (drawn with gv, orbital visualization software, ${ }^{39,40}$ isosurface value 0.08 ).

$$
\Phi_{U}=\left|a \Pi_{i} i^{i} \overline{i^{\prime \prime}}\right| .
$$

The difference between the spin-polarized MOs $i^{\prime}$ and $i^{\prime \prime}$ will introduce spin density in the whole space.

$$
\rho(r)=a(r)^{2}+\sum_{i}\left(i^{\prime}(r)^{2}-i^{\prime \prime}(r)^{2}\right) .
$$

The BS and the CI descriptions are related. One may expand both wave functions in a perturbative power expansion and compare the $\mathrm{BS}$ determinant to the $\mathrm{CI}$ wave function.

Among the excited determinants, the singly excited ones,

$$
\Phi_{i \rightarrow r}=\left|\operatorname{ari} \Pi_{k \neq i} k \bar{k}\right|
$$

and

$$
\Phi_{\bar{i} \rightarrow \bar{r}}=\left|a i \bar{r} \Pi_{k \neq i} k \bar{k}\right|,
$$

have non-zero coefficients, despite Brillouin's theorem, due to the fact that the exchange field created by the unpaired electron is different for $\alpha$ and $\beta$ spin electrons of the "core," while the Fock operator for the reference function, written as

$$
F=h+J_{a}-K_{a} / 2+\sum_{k}\left(2 J_{k}-K_{k}\right),
$$

introduces an average exchange operator $-K_{a} / 2$. The coefficients of the wave function on these two determinants are of opposite signs, as expected from spin-symmetry reasons. One may demonstrate that

$$
\left\langle\Phi_{R}|H| \Phi_{i \rightarrow r}\right\rangle=\left\langle i\left|-K_{a} / 2\right| r\right\rangle
$$

and

$$
\left\langle\Phi_{R}|H| \Phi_{\bar{i} \rightarrow \bar{r}}\right\rangle=\left\langle i\left|K_{a} / 2\right| r\right\rangle .
$$


To the first order of perturbation, the coefficients of these determinants are

$$
\begin{gathered}
c_{i r}=\left\langle i\left|-K_{a} / 2\right| r\right\rangle /\left(F_{i i}-F_{r r}\right), \\
c_{\bar{i} \bar{r}}=\left\langle i\left|K_{a} / 2\right| r\right\rangle /\left(F_{i i}-F_{r r}\right),
\end{gathered}
$$

respectively, where the energy denominator is taken as the energy difference between Fock operator diagonal elements of the hole $i$ and the particle $r$.

One may now consider the broken symmetry single determinant,

$$
\Phi_{B S}=\left|a \prod_{i} i i^{\prime \prime}\right|,
$$

where the $\alpha$ and $\beta$ spin orbitals of the core are different. To the first order of perturbation,

$$
i^{\prime}=i+\sum_{r} \frac{\left\langle i\left|K_{a} / 2\right| r\right\rangle}{F_{i i}-F_{r r}} r
$$

the mixing being opposite for $i^{\prime \prime}$.

The energy lowering brought by this orbital relaxation may be estimated to be

$$
\left\langle\Phi_{B S}|H| \Phi_{B S}\right\rangle-\left\langle\Phi_{R}|H| \Phi_{R}\right\rangle=2 \sum_{i, r} \frac{\left\langle i\left|K_{a} / 2\right| r\right\rangle^{2}}{F_{i i}-F_{r r}} .
$$

The doubly excited determinant belonging to the same space configuration,

$$
\Phi_{a \bar{i} \rightarrow r \bar{a}}=\Phi_{i r, s f}=\left|i \bar{a} r \prod_{k \neq i} k \bar{k}\right|,
$$

must be considered if one wants to have a pure doublet spin state. It represents both a spin-flip of the singly occupied orbital and a triplet excitation in the core and will hereafter be called spin-flipped. Consistently with spin-symmetry requirements, its interaction with the reference function is

$$
\left\langle\Phi_{R}|H| \Phi_{a \bar{i} \rightarrow r \bar{a}}\right\rangle=\left\langle i a\left|r_{12}^{-1}\right| a r\right\rangle=\left\langle i\left|K_{a}\right| r\right\rangle,
$$

and its coefficient is

$$
c_{a \bar{i} \rightarrow r \bar{a}}=c_{i r, s f}=\left\langle i\left|K_{a}\right| r\right\rangle /\left(F_{i i}-F_{r r}\right),
$$

where the index $s f$ indicates the spin-flipped character of the determinant. Figure 2(a) presents its first-order coefficient.

To the first-order, the spin-polarized configuration relative to $i \rightarrow r$ excitation is

$$
\Psi_{i r}^{(1)}=\left(\left|\Phi_{\bar{i} \rightarrow \bar{r}}\right\rangle-\left|\Phi_{i \rightarrow r}\right\rangle+2\left|\Phi_{a \bar{i} \rightarrow \bar{a} r}\right\rangle\right)\left\langle i\left|K_{a} / 2\right| r\right\rangle /\left(F_{i i}-F_{r r}\right),
$$

which is a pure doublet.

The energy correction brought by the double excitation is not incorporated by the BS determinant but is the major part of the spin-polarization (or spin-correlation) energy. Due to its doubleexcitation character, this spin-flip correction has a minor effect on the spin density, the operator of which is mono-electronic. In contrast, the spin-flip component is included in the CI running on the $1 \mathrm{~h}-1 \mathrm{p}$ excited configurations. This CI should a priori give a correct estimate of the coefficients of the spin-polarizing $1 \mathrm{~h}-1 \mathrm{p}$ configurations. We are aware that this truncated CI may slightly
TABLE I. Total Mulliken spin population per center in the $\mathrm{CH}_{3}$ radical.

\begin{tabular}{lcrc}
\hline \hline & $\mathrm{C}$ & $\mathrm{H}$ & \% Spin polarization \\
\hline CAS $(1,1)$ & 1.0000 & 0.0000 & 0.00 \\
1h-1p & 1.1614 & -0.0538 & 84.86 \\
2h-2p & 1.1795 & -0.0598 & 94.37 \\
3h-3p & 1.1892 & -0.0631 & 99.47 \\
4h-4p & 1.1901 & -0.0634 & 99.95 \\
CAS $(7,7)$ & 1.1902 & -0.0634 & 100.00 \\
\hline \hline
\end{tabular}

underestimate their values due to size-consistency defects, i.e. to the normalization of the wave function, as occurs for the single and double configuration interactions (SDCIs). This defect of the $1 \mathrm{~h}-1 \mathrm{p}$ CI is, however, of modest amplitude since the norm of the wave function on the $1 \mathrm{~h}-1 \mathrm{p}$ excited determinants remains small.

\section{B. A numerical surprise}

Let us consider the archetypal methyl radical $\mathrm{CH}_{3}$. All the computational details can be found in the Refs. 11-15 and in the supplementary material. In a minimal basis set, one electron occupies the $2 \mathrm{p}_{\mathrm{z}}$ orbital of carbon, and there are three doubly occupied $\mathrm{CH} \sigma$ bonding MOs and three unoccupied valence $\sigma^{*}$ antibonding MOs (Fig. 1). As spin polarization is essentially a valence effect, a valence basis CI (isomorphous to a minimal basis set) contains the main physics of the phenomenon. We next perform a series of calculations starting from the restricted function $\Phi_{R}$ and adding successively higher excitations, therefore performing successively $1 \mathrm{~h}-$ $1 \mathrm{p}, 2 \mathrm{~h}-2 \mathrm{p}, 3 \mathrm{~h}-3 \mathrm{p}, \ldots$ up to the full CI (FCI) of seven electrons in seven orbitals. Table I reports the evolution of the Mulliken atomic spin densities when the CI is pushed toward FCI and shows a systematic increase in the spin densities (positive and negative) when adding multiply excited configurations. We then concentrate our attention on the coefficients of the spin-polarizing determinants, i.e. those belonging to the $1 \mathrm{~h}-1 \mathrm{p}$ category (Table II). The computations reported in Tables I and II have been performed from the symmetry-adapted MOs, the three doubly occupied MOs are of different symmetries, as are the three virtual valence MOs, with a one-to-one correspondence between the occupied and virtual MOs (see Fig. 1) so that only three singly excited configurations appear

TABLE II. Weights of spin-polarization configurations in the $\mathrm{CH}_{3}$ radical. ${ }^{\mathrm{a}}$

\begin{tabular}{lccccc}
\hline \hline CI level & $1 \rightarrow 7$ & $2 \rightarrow 5$ & $3 \rightarrow 6$ & $C_{0}^{2}$ & $\eta(\times 100)^{\mathrm{b}}$ \\
\hline lh-1p & 0.00491 & 0.00097 & 0.00097 & 0.99314 & 0.690 \\
2h-2p & 0.00501 & 0.00108 & 0.00108 & 0.96901 & 0.740 \\
3h-3p & 0.00527 & 0.00120 & 0.00120 & 0.96809 & 0.792 \\
4h-4p & 0.00528 & 0.00120 & 0.00120 & 0.96716 & 0.794 \\
CAS(7,7) & 0.00528 & 0.00120 & 0.00120 & 0.96716 & 0.796 \\
\hline \hline
\end{tabular}

${ }^{a}$ Columns 2-4 correspond to the weights of the three spin-polarization configurations and $C_{0}^{2}$ being the weight of the reference.

${ }^{\mathrm{b}} \eta$ is the ratio of the sum of the weight of spin-polarization configurations over the weight of the reference: $\eta=\frac{1}{C_{0}^{2}} \sum C_{1 h-1 p}^{2}$. 


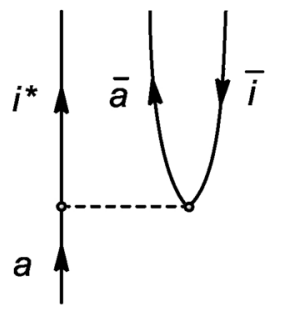

a

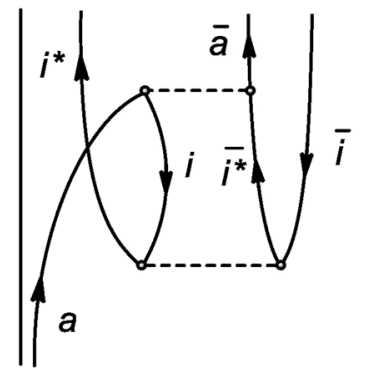

b
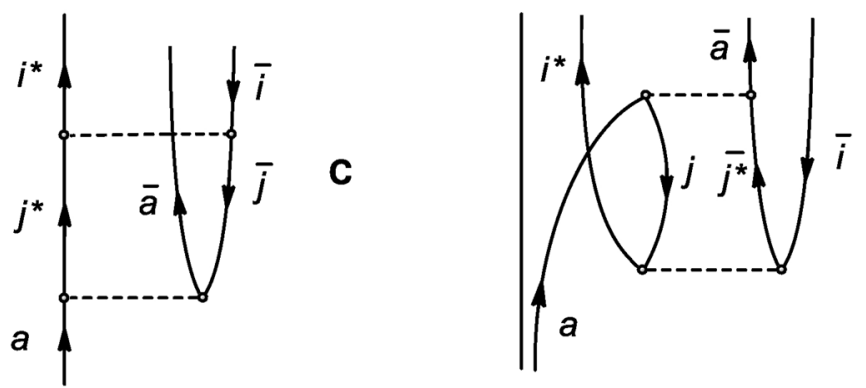

FIG. 2. Diagrammatic representation of the contributions to spin-flipped components of the $1 \mathrm{~h}-1 \mathrm{p}$ configuration. (a) First-order and (b) second-order contribution from intra-bond correlation, (c) second-order propagation between bonds, (d) second-order contribution from inter-bond correlation, (e) secondorder contribution from double spin-flip, (f) third-order contribution with double (or multiple) spin-flips and return, and (g) multiple spin-flips. Labels on propagation lines have been simplified in the last two diagrams.<smiles>CCCCCCCCCCCC</smiles>

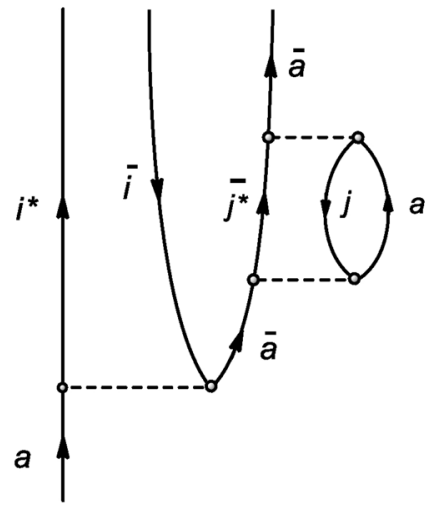

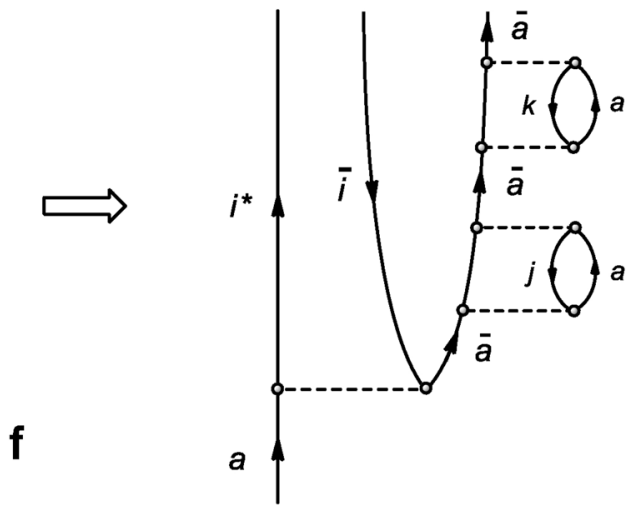

in the wave function. Let us recall that the physics of the $1 \mathrm{~h}-1 \mathrm{p}$ CI does not depend on the localized or delocalized character of the MOs.

If the first-order perturbation were sufficient to fix their amplitudes, the results of the $1 \mathrm{~h}-1 \mathrm{p}$ CI would be close to the final result.
In fact, as soon as one goes to the $2 \mathrm{~h}-2 \mathrm{p} \mathrm{CI}$, one introduces large coefficients on double excitations $\left(\sigma \rightarrow \sigma^{*}\right)^{2}$. As this brings the nondynamical correlation energy, one should expect that the weights of the $1 \mathrm{~h}-1 \mathrm{p}$ configurations decrease with respect to their values in the $1 \mathrm{~h}-1 \mathrm{p}$ CI wave function due to the introduction of these 
additional determinants. Indeed, the weight of the reference decreases from 0.993 for the $1 \mathrm{~h}-1 \mathrm{p}$ to 0.967 in the FCI, as reported in Table II. This decrease is mainly due to the double excitations, since the value for the $2 \mathrm{~h}-2 \mathrm{p}$ is 0.969 , as shown in Table II, which further illustrates how the coefficients of the leading $1 \mathrm{~h}-1 \mathrm{p}$ configurations increase when new degrees of excitations are added. For symmetry reasons, three $1 \mathrm{~h}-1 \mathrm{p}$ configurations contribute to the spin polarization. The weight of the $1 \rightarrow 7$ excitation between the fully symmetrical MOs goes from 0.0049 at the $1 \mathrm{~h}-1 \mathrm{p}$ level to 0.0053 at the FCI level. The ratio of the weight of $1 \mathrm{~h}-1 \mathrm{p}$ determinants over that of the reference increases regularly with the addition of multiple excitations.

\section{Theoretical interpretation}

This phenomenon is unexpected. It cannot be due to a normalization defect of the $1 \mathrm{~h}-1 \mathrm{p}$ CI wave function. The $2 \mathrm{~h}-2 \mathrm{p}$ CI already introduces the products of $1 \mathrm{~h}-1 \mathrm{p}$ excitations but is largely dominated by double excitations, and the relative weight of $1 \mathrm{~h}-1 \mathrm{p}$ determinants still increases. The reason for this increase is to be searched in the interactions between $1 \mathrm{~h}-1 \mathrm{p}$ determinants and $2 \mathrm{~h}-2 \mathrm{p}$ excited determinants, which are obtained directly either by a double excitation on the reference determinant or by a second single excitation on the top of the first one. Hereafter, we shall focus on the spin-flip components of the $1 \mathrm{~h}-1 \mathrm{p}$ configurations, since they have the major coefficient and since their diagrammatic representation [Fig. 2(a)] is simpler than for the strictly singly excited components.

\section{Interplay between intra-bond correlation and spin polarization}

The first mechanism is the interaction between correlation and spin polarization. One may illustrate it in the case of $\mathrm{CH}_{3}$. Both non-dynamical correlation and spin polarization take place among valence MOs, and it is worth considering localized valence bonding $\mathrm{MOs}$, one per $\mathrm{CH}$ bond, with their localized antibonding counterparts. One should note that in this molecule, in a minimal basis set, there is no virtual orbital of $a$ symmetry and no double excitation involving the $a$ orbital in the wave function. The correlation effects essentially concern intra-bond double excitation. Let us consider a $\left(\sigma \rightarrow \sigma^{*}\right)^{2}$ intra-bond double excitation from the localized MO $i$ to its antibonding counterpart $i^{*}$, leading to

$$
\Phi_{i \bar{i} \rightarrow i^{*} \bar{i}^{*}}=\left|a i^{*} \bar{i}^{*} \Pi_{k \neq i} k \bar{k}\right| .
$$

The coefficient of this doubly excited determinant is necessarily negative,

$$
c_{i \bar{i} \rightarrow i^{\star} i^{\star}}=\frac{K_{i i^{*}}}{2\left(F_{i i}-F_{i^{\star} i^{*}}\right)}<0 .
$$

By convention, we shall give a positive coefficient of the $\sigma^{*}$ MOs on carbon hybrids. The interaction between this doubly excited determinant and the spin-flipped $1 \mathrm{~h}-1 \mathrm{p}$ one is

$$
\left\langle\Phi_{i \bar{i} \rightarrow i^{*} i^{*}}|H| \Phi_{i i^{*}, s f}\right\rangle=\left\langle i^{*}\left|-K_{a}\right| i\right\rangle .
$$

Therefore, the second-order contribution to the coefficient of $\left|\Phi_{i i^{*}, s f}\right\rangle$, resulting from its interaction with the doubly excited determinant, is,

$$
c_{\bar{i}^{*}, s f}^{(2)}=c_{i \bar{i} \rightarrow i^{*}} \frac{\left\langle i^{*}\right.}{\left(i_{i i}-F_{i^{*} i^{*}}\right)},
$$

of the same sign as the first-order contribution. To the second order of perturbation,

$$
c_{\overline{i i}^{*}, s f}^{(1+2)}=c_{i i^{*}, s f}^{(1)}\left(1-c_{i \bar{i} \rightarrow i^{*} i^{*}}\right),
$$

since $c_{a \bar{i} \rightarrow i^{*} \bar{a}}^{(1)}=c_{i i^{*}, s f}=\left\langle i\left|K_{a}\right| i^{*}\right\rangle /\left(F_{i i}-F_{i^{*} i^{*}}\right)$.

As illustrated in Fig. 2(b), the intra-bond double excitation enhances the coefficient of the spin-polarizing single determinants. There is definitely an interference between spin polarization and electronic correlation.

\section{Propagation of spin polarization and effect of inter- bond double excitations}

Then, one may wonder whether spin polarization propagates from one bond to the other. The interaction between the $1 \mathrm{~h}-1 \mathrm{p}$ determinant relative to bond $j, \Phi_{j j^{*}, s f}=\left|i \overline{i j} \bar{a} j{ }^{*} \Pi_{k \neq i, j} k \bar{k}\right|$ with $\Phi_{i i^{*}, s f}$, relative to bond $i$, happens to be $-\left(i j, i^{*} j^{*}\right)$ so that

$$
c_{j j^{*}, s f}^{(2)}=\frac{-\left(i j, i^{*} j^{*}\right)}{\left(F_{j j}-F_{j^{*} j^{*}}\right)}\left(c_{i i^{*}, s f}\right) .
$$

The bi-electronic integral $-\left(i j, i^{*} j^{*}\right)$ essentially involves the on-site exchange integral between carbon hybrid orbitals of two $\mathrm{CH}$ bonds, and it is positive under our convention of sign in the antibonding MOs. Therefore, this propagative contribution is of the same sign as the first-order contribution. This process, which remains in the space of the $1 \mathrm{~h}-1 \mathrm{p}$ configurations, is illustrated in Fig. 2(c).

It possesses a counterpart passing through an inter-bond double excitation on bonds $i$ and $j$, illustrated in Fig. 2(d). The process involves the determinant

$$
\Phi_{i \bar{j} \rightarrow j^{*} \bar{i}^{*}}=\left|a j^{*} \overline{i j i^{\star}} \Pi_{k \neq i, j} k \bar{k}\right|,
$$

the coefficient of which is

$$
c_{i \overline{j \rightarrow j^{*} i^{*}}}^{(1)}=\frac{\left(i j^{*}, j i^{*}\right)}{\left(F_{i i}+F_{j j}-F_{i^{*} i^{*}}-F_{j^{*} j^{*}}\right)} .
$$

Its interaction with $\Phi_{i i^{*}, s f}$ is equal to $\left\langle j^{*}\left|K_{a}\right| j\right\rangle$ so that the second-order contribution to the $\mathrm{i} \rightarrow \mathrm{i}^{*}$ spin-flipped determinant is

$$
c_{i \rightarrow i^{*}, s f}^{(2)}=-\frac{\left\langle j^{*}\left|K_{a}\right| j\right\rangle\left(i j^{*}, j i^{*}\right)}{\left(F_{i i}-F_{i^{*} i^{*}}\right)\left(F_{i i}+F_{j j}-F_{i^{*} i^{*}}-F_{j^{*} j^{*}}\right)} .
$$

This process, illustrated in Fig. 2(d), gives a contribution to the spin polarization passing through a preliminary double excitation and may contribute to the enhancement of the spin-polarizing singly excited determinants when going from the $1 \mathrm{~h}-1 \mathrm{p}$ CI to the $2 \mathrm{~h}-2 \mathrm{p}$ level. It confirms the positive interference between spin polarization and correlation. 


\section{Multiple single excitations}

The last mechanism may be sketched as "reference $\rightarrow$ single excitation ( $1 \mathrm{~h}-1 \mathrm{p}$ determinant) $\rightarrow$ second single excitation $(2 \mathrm{~h}$ $-2 \mathrm{p}$ determinant $) \rightarrow$ single de-excitation ( $1 \mathrm{~h}-1 \mathrm{p}$ determinant)." One may consider two $1 \mathrm{~h}-1 \mathrm{p}$ excitations, namely, $i \rightarrow i^{*}$ and $j \rightarrow j^{*}$. If one has performed first the spin-flipped excitation relative to bond i, $a_{\bar{a}}^{+} a_{i^{*}}^{+} a_{a} a_{\bar{i}}$, leading to $\Phi_{\bar{i} a \rightarrow \bar{a} i^{*}}=\left|\bar{a} i^{*} i \prod_{k \neq i} k \bar{k}\right|$, bond $i$ is in $m_{s}=1$ triplet state. One may then apply a new spin-flip excitation relative to bond $j$, and through the operator $a_{a}^{+} a_{\bar{j}^{\star}}^{+} a_{\bar{a}} a_{j}$, one reaches the same 2h-2p determinant $\Phi_{\overline{i j \rightarrow j^{*} i^{*}}}=\left|a j^{*} \overline{i j} \overline{i^{*}} \Pi_{k \neq i, j} k \bar{k}\right|$ in which bonds $i$ and $j$ are in $m_{s}=1$ and $m_{s}=-1$ triplet states, respectively. The interaction between the singly excited and the doubly excited determinants again implies the $K_{a}$ operator,

$$
\left\langle\Phi_{\bar{i} a \rightarrow \bar{a} i^{*}}|H| \Phi_{i \bar{j} \rightarrow j^{*} i^{*}}\right\rangle=\left\langle j\left|K_{a}\right| j^{*}\right\rangle .
$$

From the doubly excited determinant, one may return to the $1 \mathrm{~h}-1 \mathrm{p}$ $i \rightarrow i^{*}$ spin-flip determinant through the same interaction so that its coefficient is increased by the action of the spin-polarization on the other bonds,

$$
c_{\bar{i} \rightarrow \bar{i}^{*}, s f}^{(1+3)}=c_{\bar{i} \rightarrow i^{*}, s f}\left(1+\sum_{j \neq i} \frac{\left\langle j\left|K_{a}\right| j^{*}\right\rangle^{2}}{\left(F_{i i}-F_{i^{*} i^{*}}\right)\left(F_{i i}+F_{j j}-F_{i^{*} i^{*}}-F_{j^{*} j^{*}}\right)}\right) .
$$

The action of the spin-polarizing operator acting on bonds $j$ amplifies the coefficient of the spin-polarized configuration on bond $i$. The process, illustrated in Fig. 2(e), follows the sequences

$$
\Phi_{0} \rightarrow \Phi_{i i^{*}, s f} \rightarrow \Phi_{\left(\overline{i j} \rightarrow j^{*} \bar{i}^{*}\right)} \rightarrow \Phi_{i i^{*}, s f}
$$

In terms of configurations, this may be seen as belonging to the process

$$
\Phi_{0} \rightarrow \Psi_{i i^{*}}^{(1)} \rightarrow\left(i j \rightarrow i^{*} j^{*}\right)_{\text {doubly-excited }} \rightarrow \Psi_{i i^{*}}^{(1)},
$$

which necessarily enhances the coefficients of the $1 \mathrm{~h}-1 \mathrm{p}$ configuration. One should remark that the same back-and-forth spin-flip concerning the $j-j^{*}$ spin-flip excitations on top of the previous spinflip excitation of bond $i$ may be repeated, introducing higher-order terms,

$$
\begin{aligned}
c_{\bar{i} \rightarrow \bar{i}, s f}^{(1+3)}= & c_{\bar{i} \rightarrow \bar{i} *, s f}\left(1+\sum_{j \neq i} \frac{\left\langle j\left|K_{a}\right| j^{*}\right\rangle^{2}}{\left(F_{i i}-F_{i^{*} i^{*}}\right)\left(F_{i i}+F_{j j}-F_{i^{*} i^{*}}-F_{j^{*} j^{*}}\right)}\right. \\
& \left.+\left(\sum_{j \neq i} \frac{\left\langle j\left|K_{a}\right| j^{*}\right\rangle^{2^{\prime}}}{\left(F_{i i}-F_{i^{*} i^{*}}\right)\left(F_{i i}+F_{j j}-F_{i^{*} i^{*}}-F_{j^{*} j^{*}}\right)}\right)^{2}+\cdots\right) .
\end{aligned}
$$

These corrections may be expressed as a change of the excitation energy to the $i \rightarrow i^{*}$ spin-flip configuration,

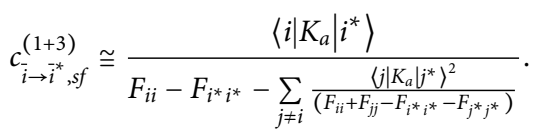

The summation of these excitations results in a reduction in the effective excitation energy from the reference to the spin-polarizing 1h-1p configuration.
Moreover, one might also introduce a $k \rightarrow k^{*}$ spin-flip excitation on top of the doubly excited determinant $\Phi_{\left(\overline{i j} \rightarrow j^{*} i^{*}\right)}$, which leads to a triply excited determinant, lowering the effective energy of this determinant. The mechanism is illustrated in Fig. 2(f). It represents some kind of entangled cascade of spin polarizations. The denominators relative to the multiple excitations increase, but the number of combinations of the successive events also increases. If one remains in the $\mathrm{CH}_{3}$ problem, the number of one-spin-flip processes, in which $a$ becomes spin down, is 3 . The number of two-spin-flips, in which $a$ returns to spin up, is 6 . If one calls $\mu$ the integrals $\left\langle\mathrm{i}\left|\mathrm{K}_{\mathrm{a}}\right| \mathrm{i}^{*}\right\rangle$, and $\Delta$ the excitation energy on a bond, the second-order energy correction is $y=3 \mu^{2} / \Delta$. The fourth-order corrections coming from the double spin-flips is $6 \mu^{4} / 2 \Delta^{3}=3 \mu^{4} / \Delta^{3}$. One may calculate the effect of the three-spin-flip configurations (which belong to the $3 \mathrm{~h}-3 \mathrm{p}$ subspace). Their number is 3 , and their coefficient is $\mu^{3} / 3 \Delta^{3}$. Their effect on the energy is $\mu^{5} / \Delta^{4}$, and their effect on the coefficients of the $1 \mathrm{~h}-$ $1 \mathrm{p}$ configurations is $\mu^{4} / 3 \Delta^{4}$. This analysis may explain the observed increase in the $1 \mathrm{~h}-1 \mathrm{p}$ configuration when passing from the $2 \mathrm{~h}-2 \mathrm{p}$ CI to the $3 h-3 p$ CI.

It is important to note that the second spin-flip operator $a_{a}^{+} a_{\tilde{j}^{*}}^{+} a_{\bar{a}} a_{j}$ does not act on the reference single determinant. Figure 2(f) illustrates entirely connected processes. This connectivity is in contrast with the typical single-reference correlation problem where the leading contributions to the quadruply excited determinant are products of disjoint double excitation coefficients. Here, the leading term to the double-spin-flip determinants is intrinsically connected and requires the action of an excitation that does not act on the reference. As will be discussed later, this questions the possibility to treat the spin-polarization phenomenon from a single reference coupled cluster expansion.

\section{Broken-symmetry transcription}

In order to understand why double excitation results in an enhancement of the coefficients of singly excited ones, it is worth using localized bonding and antibonding MOs on the three $\mathrm{CH}$ bonds, and consider the excitation on bond $j$ after a preliminary spin polarization of bond $i$. We shall first follow the physics involved in BS treatments. The single excitation on bond $i$ may be seen as a spin-dependent revision of the MOs leading to the BS determinant,

$$
\Phi_{B S}(i)=\left|a j \overline{j i} i^{\bar{i}}\right|,
$$

where $i^{\prime}$ has a larger amplitude on the carbon atom than $i$ due to the exchange attraction by the spin on atomic orbital $a$, while the trend change is opposite for $i^{\prime \prime}$. The orbital rotation essentially involves the antibonding orbital of the same bond,

$$
i^{\prime}=i+\lambda_{i} i^{*}
$$

with

$$
\lambda_{i}=\frac{\left\langle i\left|-K_{a} / 2\right| i^{*}\right\rangle}{F_{i i}-F_{i^{*} i^{*}}} .
$$

If one gives a positive coefficient on the carbon hybrid $\mathrm{AO}$ in $i^{*}$, this coefficient is positive and scales as

$$
\lambda_{i}=-\frac{K_{d a} / 4}{F_{i i}-F_{i^{*} i^{*}}},
$$


where $K_{d a}$ is the intra-atomic exchange integral between $2 p_{z}$ orbital $a$ and the $s p^{2}$ hybrid orbital on carbon involved in bond i. On the contrary,

$$
i^{\prime}=i-\lambda_{i} i^{*} \text {. }
$$

This $\mathrm{MO}$ has a smaller amplitude on the carbon hybrid AO.

Polarizing now the MOs of bond $j$ through $j \rightarrow j^{*}$ excitation of a spin, one gets

$$
\left\langle a j^{*} \bar{j} i^{\prime} \overline{i^{\prime}}|H| a j \overline{j i} \bar{i}^{\prime} \overline{i^{\prime}}\right\rangle=\left\langle j^{*}\left|-K_{a} / 2+J_{i^{\prime}}+J_{i^{\prime}}-2 J_{i}-K_{i^{\prime}}+K_{i}\right| j\right\rangle .
$$

One may show that

$$
\left\langle j^{*}\left|J_{i^{\prime}}+J_{i^{\prime}}-2 J_{i}\right| j\right\rangle=0
$$

and hence,

$$
\left\langle a j^{*} \overline{j i} i^{\prime} \overline{i^{\prime}}|H| a j \bar{j} i^{\prime} \overline{i^{\prime}}\right\rangle=\left\langle j^{*}\left|-K_{a} / 2-K_{i^{\prime}}+K_{i}\right| j\right\rangle .
$$

One may develop the quantity $\left\langle j^{*}\left|K_{i^{\prime}}-K_{i}\right| j\right\rangle$ in terms of the coefficients of the orbitals on the unique non-negligible atomic integral, namely, the exchange integral $k_{p i}$ j between the carbon hybrids $p_{i}$ and $p_{j}$ involved in bonds $i$ and $j$, respectively,

$$
\left\langle j^{*}\left|K_{i^{\prime}}-K_{i}\right| j\right\rangle \cong k_{p i, p j} c_{j^{*} p_{j}} c_{j p_{j}}\left(c_{i^{\prime}, p_{i}}^{2}-c_{i, p_{i}}^{2}\right) .
$$

Due to the larger amplitude of $i^{\prime}$ on the carbon atom,

$$
\left\langle j^{*}\left|-K_{i^{\prime}}+K_{i}\right| j\right\rangle<0,
$$

$\left\langle j^{*}\left|K_{i^{\prime}}-K_{i}\right| j\right\rangle$ has the same sign as $\left\langle j^{*}\left|-K_{a} / 2\right| j\right\rangle$. Therefore, the amplitude of the interaction responsible for the spin polarization of bond $j$ has been increased by the preliminary spin polarization of bond $i$,

$$
\left|\left\langle a j^{*} \bar{j} i^{\prime} \overline{i^{\prime \prime}}|H| a j \overline{j i} i^{\prime} \overline{i i^{\prime \prime}}\right\rangle\right|>\left|\left\langle a j^{*} \overline{j i} \bar{i}|H| a j \overline{j i} \bar{i}\right\rangle\right| .
$$

One has the same inequality for the $j \rightarrow j^{*}$ excitation of $\beta$ spin. Once the $\alpha$ spin electron of the first $\mathrm{CH}$ bond has been pushed closer to the a spin unpaired electron, it reinforces the exchange field acting on the a spin electron of the second $\mathrm{CH}$ bond.

One should remember that the dominant valence bond determinant of $\mathrm{CH}_{3}$ is $\left|a \cdot p_{1} p_{2} p_{3} \bar{h}_{1} \bar{h}_{2} \bar{h}_{3}\right|$, which satisfies the neutrality of the atoms and Hund's rule and corresponds to the correct dissociation limit into atoms in their ground states. The spin polarization mechanism increases the weight of this VB component.

\section{Conclusive remark}

The above-discussed mechanisms contribute to the cooperative effect observed in the numerical calculations. It is difficult to assess the respective roles of the various mechanisms evoked in this discussion, namely, the interference between correlation and spin polarization, and the cooperative character of the spin polarization. Nevertheless, looking at the increase in the coefficients of spinpolarization singly excited determinants when going from the $2 \mathrm{~h}-2 \mathrm{p}$ $\mathrm{CI}$ to the $3 \mathrm{~h}-3 \mathrm{p} \mathrm{CI}$, the cooperative mechanisms seem more likely. As will be shown in Sec. III, the same phenomenon takes place in diradicals.

\section{A DRAMATIC SPIN POLARIZATION IN A SINGLET DIRADICAL}

Spin-polarization is also of major importance in diradicals, as qualitatively illustrated by the singlet character of the ground state of the $90^{\circ}$ twisted ethylene $e^{5}$ and quantitatively crucial in CI calculations of magnetic couplings. ${ }^{16-18}$

\section{A. Choice of paradigmatic problem}

Diradicals, in principle, exhibit two singly occupied orbitals. This picture is not problematic for ferromagnetic systems, the ground state of which is of triplet character. When the ground state is a singlet, there is no absolute difference between diradicals and closed shell systems, and a lot of criteria have been proposed to identify diradicals or measure the diradical character. ${ }^{19,20}$ For an experimentalist, the key property is the magnetic susceptibility, hence the amplitude of the singlet-triplet gap, but actually one may identify several leading contributions to this energy difference. ${ }^{16-18}$ These are essentially a direct exchange (always ferromagnetic, i.e. in favor of the triplet), the so-called antiferromagnetic kinetic exchange, which reflects the stabilizing effect of the ionic $\mathrm{VB}$ component of the singlet state, and a spin-polarization mechanism, the sign of which depends on the molecular architecture. To study the latter contribution, one may select diradicals in which kinetic exchange is zero for symmetry reasons, the magnetic preference being essentially governed by the spin-polarization mechanism. Such is the case of conjugated molecules where a double bond has been twisted by $90^{\circ}$, the simplest one being twisted ethylene. ${ }^{5,6}$ We illustrate the occurrence of a spin-polarization collective effect in a singlet diradical, the square carbomer derivative of cyclobutadiene, $\mathrm{C}_{12} \mathrm{H}_{4}$ scheme (1). ${ }^{21}$ The relative energies of $D_{2 h}$ rectangular closed-shell singlet and of $D_{4 h}$ square open-shell geometry represent another topic, which will be discussed elsewhere.

The spin-polarization mechanism is here particularly dramatic as it is the only physical effect stabilizing the singlet below the triplet in the $D_{4 h}$ geometry. The system has $12 \pi$ electrons in $12 \pi$ valence orbitals. In its square geometry, the molecule presents two nonbonding MOs, which are of zero energy in Hückel or Hubbard models. The molecule is "alternant" (or "bipartite" in the language of physicists) in that one of two colors may be attributed to each site in such a manner that a "red" atom is linked to two "blue" atoms and vice versa (Fig. 3, left). The two non-bonding orbitals, labeled $a$ and $b$, can be localized on red and blue color sites, respectively. Putting ten $\pi$ electrons in five $\pi$ doubly occupied orbitals defines a two-open shell singlet configuration $\Phi_{S}=\left|(a \bar{b}+b \bar{a}) \Pi_{k} k \bar{k}\right| / \sqrt{2}$. The two orbitals $a$ and $b$, appearing in Fig. 3 (right), may be localized on red and blue atoms, respectively, under a $\pi / 4$ rotation of the orbitals $\mathrm{a}^{\prime}$ and $\mathrm{b}^{\prime}$ of Fig. 4, and may be written as

$$
\begin{gathered}
|a\rangle=\sum_{p=0,5}|2 p+1\rangle(-1)^{p} / \sqrt{6}, \\
|b\rangle=\sum_{p=1,6}|2 p\rangle(-1)^{p} / \sqrt{6} .
\end{gathered}
$$

According to the "mirror theorem," 22,23 each doubly occupied $\pi$ orbital $k$ is associated with a $\pi^{*}$ virtual orbital $k^{*}$ such that the coefficients of the red color (say, the odd-numbered) carbon atoms are 
<smiles>C1=C=C=C=CC#CC=C=C=1</smiles>

$D_{2 h}$

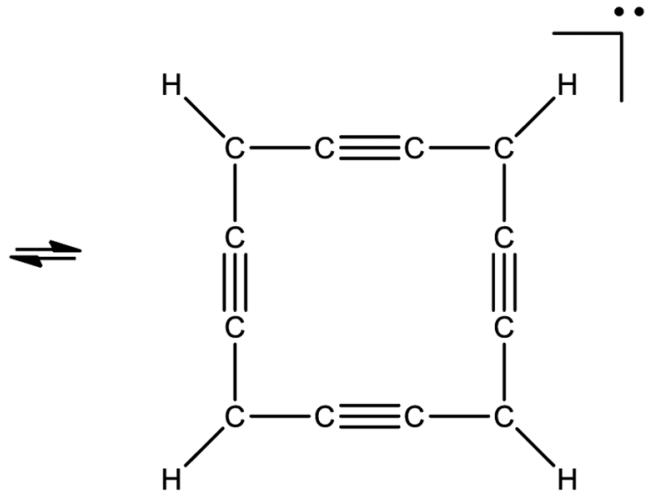

$D_{4 h}$<smiles>C1=C=C=CC=C=CC=C=C=1</smiles>

$D_{2 h}$

SCHEME 1. Interconversion pathway between equivalent $\mathrm{D} 2 \mathrm{~h}$ geometries of carbo-cyclobutadiene

the same in $k$ and $k^{*}$ orbitals, while the coefficients of the other color change their signs,

$$
\begin{gathered}
C(k, 2 p+1)=C\left(k^{*}, 2 p+1\right), \\
C(k, 2 p)=-C\left(k^{*}, 2 p\right) .
\end{gathered}
$$

The CASSCF orbitals are presented in Fig. 4 and follow the mirror rule.

Regarding the interaction between the doubly occupied and empty MOs, the Fock operator associated with the reference configuration is

$$
F=h+J_{a}-K_{a} / 2+J_{b}-K_{b} / 2+\sum_{k} 2 J_{k}-K_{k} .
$$

In one of the two single determinants involved in $\Phi_{S}$, the a spin unpaired electron is on red atoms, the $\beta$ spin unpaired electron being on blue atoms, the repartition of the spins being opposite in the other component of the reference. The two unpaired electrons induce a fluctuating exchange field on the "core" electrons. To the first order, the spin-polarization process goes through $1 \mathrm{~h}-1 \mathrm{p}$ excited configurations, singly excited either with respect to one of the components of $\Phi_{S}$, such as

$$
\Phi_{\bar{k} \rightarrow \bar{r}, a \bar{b}}=\left|\Pi_{i \neq k} \bar{i} \bar{k} \bar{r} a \bar{b}\right| \text { and } \Phi_{k \rightarrow r, a \bar{b}}=\left|\Pi_{i \neq k} \bar{i} r \bar{k} a \bar{b}\right|,
$$

or with their spin-flipped counterparts,

$$
\Phi_{k \rightarrow \bar{r},}^{S F}=\left|\Pi_{i \neq k} i \bar{i} \bar{k} \bar{r} a b\right| \text { and } \Phi_{\bar{k} \rightarrow r,}^{S F}=\left|\Pi_{i \neq k} i \bar{i} k r \bar{a} \bar{b}\right| .
$$

To the second order of perturbation, the resulting energy of these interactions is given by

$$
{ }^{1} E_{S P}^{(2)}=\sum_{k, r} \frac{3 / 2\left\langle k\left|K_{a}-K_{b}\right| r\right\rangle^{2}}{F_{k k}-F_{r r}} .
$$

It is interesting to use a Hubbard approximate Hamiltonian,

$$
H=\sum_{p, q} t_{p q} a_{p}^{+} a_{q}+\sum_{p} U a_{p}^{+} a_{\bar{p}}^{+} a_{\bar{p}} a_{p}
$$
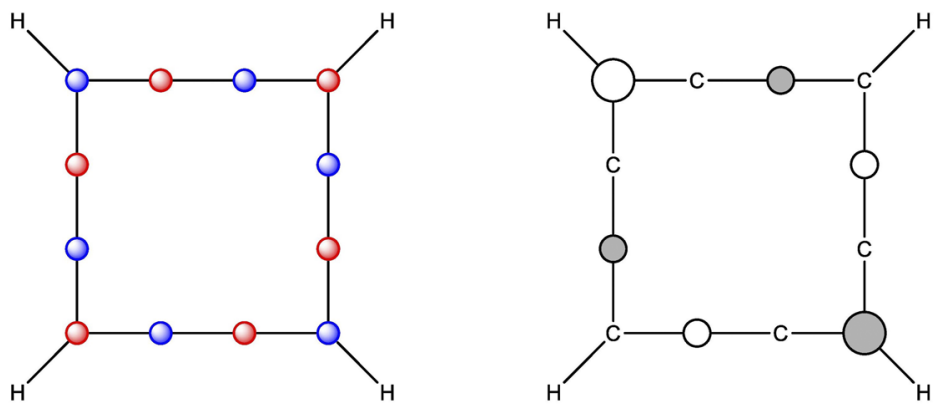

a

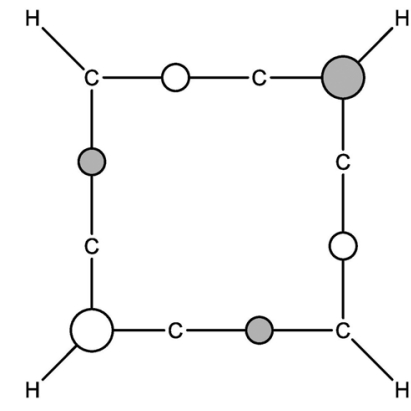

b

FIG. 3. Carbo-cyclobutadiene $\mathrm{C}_{12} \mathrm{H}_{4}$. (Left) Sketching the alternant character. (Right) The two most-localized singly occupied orbitals $a$ and $b$. 


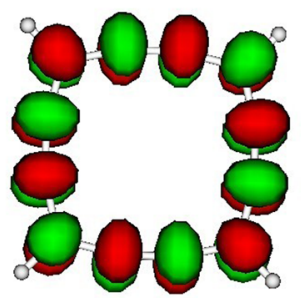

12

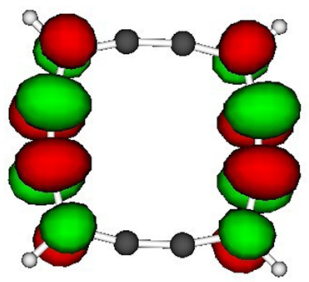

11

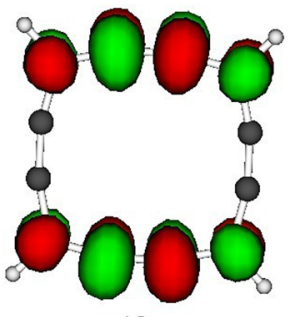

10

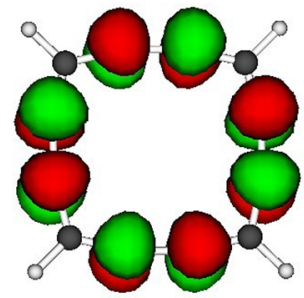

9

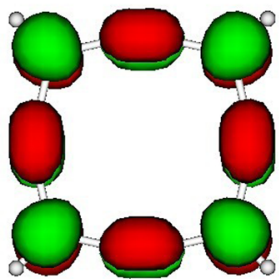

8

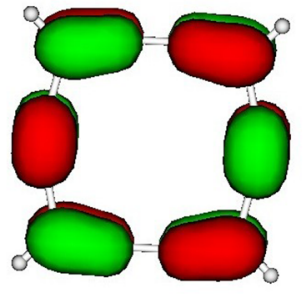

a'

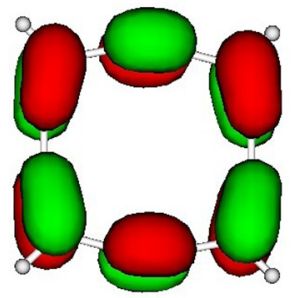

b'

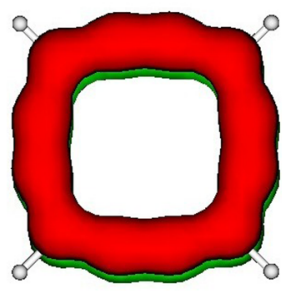

1

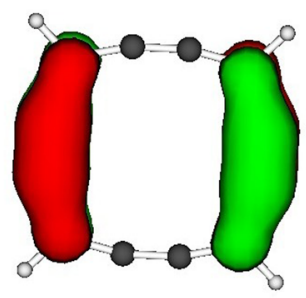

2

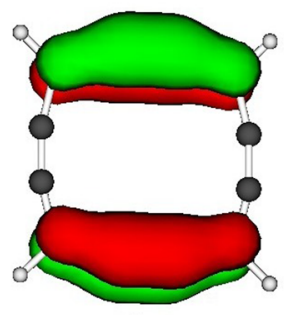

3

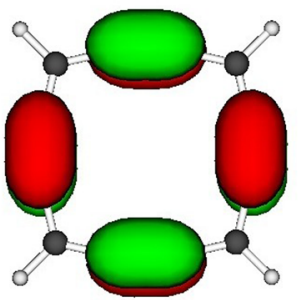

4

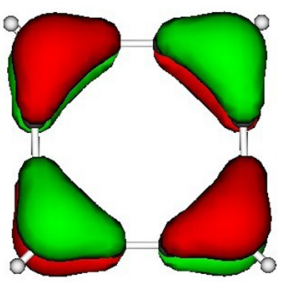

5

FIG. 4. Molecular orbitals of carbo-cyclobutadiene $\mathrm{C}_{12} \mathrm{H}_{4}$ (drawn with gv, orbital visualization software, ${ }^{39,40}$ isosurface value 0.04 ).

to identify the major contributions to this sum,

$$
\left\langle k\left|K_{a}-K_{b}\right| r\right\rangle=U \sum_{p} C_{p k} C_{p r}\left(C_{p a}^{2}-C_{p b}^{2}\right) .
$$

In this problem, for symmetry reasons, $C_{p a}^{2}=1 / 6$ if $p$ is odd and $C_{p a}^{2}$ $=0$ if $p$ is even, with the reverse relation for the $b$ orbital, so that

$$
\left\langle k\left|K_{a}-K_{b}\right| r\right\rangle=U\left(\sum_{p, o d d} C_{p k} C_{p r} / 6-\sum_{p, e v e n} C_{p k} C_{p r} / 6\right) .
$$

If $r=i^{*}, \neq k^{*}$,

$$
\left\langle k\left|K_{a}-K_{b}\right| r\right\rangle=U\left\langle k \mid i^{*}\right\rangle / 6=0,
$$

the contribution of this excitation to the spin polarization energy is zero. Spin polarization reduces to paired $k \rightarrow k^{*}$ excitations between mirror orbitals and is a simple sum of contributions running on excitations between mirror orbitals,

$$
{ }^{1} E_{S P}^{(2)}=\sum_{k} \frac{3 / 2\left\langle k\left|K_{a}-K_{b}\right| k^{*}\right\rangle^{2}}{F_{k k}-F_{k \star k *}} .
$$

This is the perturbative expression of the effect of the $1 \mathrm{~h}-1 \mathrm{p}$ excitations. The coefficients of the corresponding singly excited determinants are, for instance,

$$
C_{(k \rightarrow k *) a \bar{b}}^{(1)}=\sum_{k, r} \frac{1 / 2\left\langle k\left|K_{a}-K_{b}\right| k^{*}\right\rangle^{2}}{F_{k k}-F_{k * k *}} .
$$

\section{B. Numerical study}

The computation is performed in a non-minimal basis set of atomic orbitals. ${ }^{15}$ A preliminary full valence $\pi \operatorname{CASSCF}(12,12)$ is performed, which defines optimal five bonding, two non-bonding, and five antibonding MOs. These orbitals are used in the following truncated CI calculations, the starting description is the $\operatorname{CAS}(2,2)$ function, and one adds progressively $1 \mathrm{~h}-1 \mathrm{p}, 2 \mathrm{~h}-2 \mathrm{p}, \ldots$, up to $5 \mathrm{~h}-5 \mathrm{p}$, compared to full valence $\pi$ CAS.

The surprising result is that the largest coefficients, beyond those of the two reference determinants, belong to the $1 \mathrm{~h}-1 \mathrm{p}$ class, whatever the CI level. Table III reports the weights of the reference 
TABLE III. Weight of the leading configurations in the $\mathrm{C}_{12} \mathrm{H}_{4}$ diradical at different $\mathrm{Cl}$ levels.

\begin{tabular}{|c|c|c|c|c|c|c|}
\hline CI level & lhlp & $2 \mathrm{~h} 2 \mathrm{p}$ & $3 h 3 p$ & $4 h 4 p$ & $5 h 5 p$ & Full CAS \\
\hline \multicolumn{7}{|c|}{ Weights and ratios to reference (\%) } \\
\hline Reference & 0.8675 & 0.7936 & 0.7443 & 0.7259 & 0.7215 & 0.7207 \\
\hline $1 \rightarrow 12$ & $0.0106(1.2 \%)$ & $0.0082(1.0 \%)$ & $0.0096(1.3 \%)$ & $0.0094(1.3 \%)$ & $0.0094(1.3 \%)$ & $0.0094(1.3 \%)$ \\
\hline $2 \rightarrow 11$ & $0.0145(1.7 \%)$ & $0.0115(1.4 \%)$ & $0.0134(1.8 \%)$ & $0.0132(1.8 \%)$ & $0.0132(1.8 \%)$ & $0.0132(1.8 \%)$ \\
\hline $3 \rightarrow 10$ & $0.0145(1.7 \%)$ & $0.0115(1.4 \%)$ & $0.0134(1.8 \%)$ & $0.0132(1.8 \%)$ & $0.0132(1.8 \%)$ & $0.0132(1.8 \%)$ \\
\hline $4 \rightarrow 9$ & $0.0274(3.2 \%)$ & $0.0242(3.0 \%)$ & $0.0286(3.8 \%)$ & $0.0283(3.9 \%)$ & $0.0284(3.9 \%)$ & $0.0284(3.9 \%)$ \\
\hline $5 \rightarrow 8$ & $0.0627(7.2 \%)$ & $0.0602(7.6 \%)$ & $0.0715(9.6 \%)$ & $0.0714(9.8 \%)$ & $0.0717(9.9 \%)$ & $0.0716(9.9 \%)$ \\
\hline Largest $2 \mathrm{~h}-2 \mathrm{p}$ & $\ldots$ & 0.0056 & 0.0067 & 0.0077 & 0.0077 & 0.0078 \\
\hline
\end{tabular}

configuration and those of the most important $1 \mathrm{~h}-1 \mathrm{p}$ determinants. This prevalence of single excitations never happens in closed-shell problems, where doubly excited configurations dominate the extrareference part of the wave function. Calling HDOMO the highest doubly occupied MO and LDUMO the virtual MO of lowest energy, the largest coefficients concern the HDOMO $\rightarrow$ LDUMO $(5 \rightarrow 8)$ spin-polarization configuration.

As expected from analytical derivation, the other $1 \mathrm{~h}-1 \mathrm{p}$ configurations are $k \rightarrow k^{*}$, with decreasing weight when $k$ and $k^{*}$ go further from the Fermi level, reflecting the increasing excitation energy appearing in denominators. An intriguing and counterintuitive outcome is the increase in the ratio of the weight of $1 \mathrm{~h}-1 \mathrm{p}$ configurations to that of the reference function when multiple excitations are introduced in the CI. The only exceptions concern the 1 $\rightarrow 12,2 \rightarrow 11$, and $3 \rightarrow 10$ excitations when going from the $1 \mathrm{~h}-1 \mathrm{p}$ to the $2 \mathrm{~h}-2 \mathrm{p}$ level. This step introduces large contributions from the correlation double excitations, which result in a 0.07 decrease in the weight of the reference. Beyond this step, the increase in the weight of the $1 \mathrm{~h}-1 \mathrm{p}$ configurations is regular. The effect is far from being negligible; for instance, for the HDOMO-LDUMO excited configuration, this ratio goes from 0.063 for the $1 \mathrm{~h}-1 \mathrm{p}$ step to 0.072 for the full CI, representing a $14 \%$ increase.

These numbers confirm the collective and cooperative character of the spin polarization phenomenon. It is possible to perform a rationalization similar to the one proposed in Sec. II C, showing that the interaction relative to excitation $i \rightarrow i^{*}$ is larger when $k \rightarrow k^{*}$ has already been performed. The demonstration is analytic, assuming a Hubbard Hamiltonian and taking benefit of the "mirror theorem." 22,23 Starting from $\Phi_{a \bar{b}}=\left|(a \bar{b}) \Pi_{k} k \bar{k}\right|$, where the $\alpha$-spin unpaired electron is on odd numbered atoms and the $\beta$-spin unpaired electron is on even ones, the polarization on MO $i$, leading to $i^{\prime}$, increases the coefficients on the odd-numbered sites and decreases them on the even ones. It is the opposite effect for the $\beta$ spin electron of MO $i$, polarized into $i^{\prime \prime}$. The already increased concentration of the $\alpha$ spin electron on the odd-numbered sites and of the $\beta$ spin electrons on the even ones reinforces the strength of spin polarization of the electrons of $\mathrm{MO} k$.

Notice that in alternant systems, the single determinants of major coefficients in the wave function are the so-called Néel functions, i.e., VB distributions where electrons are distributed one per site, with spin alternation on atoms of both colors. This is partly due to anti-symmetrization itself ${ }^{24}$ and partly due to electron repulsion. $^{25}$ It can be demonstrated whatever the delocalization/repulsion ratio, and it is at the root of Ovchinnikov's rule. ${ }^{26}$ Symmetry breaking of the Hartree-Fock single determinant pushes the wave function in the direction of the (or a) Néel determinant, and the collective character of spin polarization reflects this impact of a global and cooperative spin-ordering effect. In full generality, one may demonstrate that the valence bond determinants of major coefficients in the correlated wave function are those which have parallel spins on the atomic orbitals of the same atom and present spin alternation between neighbor atoms. This is true for conjugated or saturated hydrocarbons, for organic or inorganic molecules. ${ }^{27,28}$

It is possible to translate the theoretical interpretation proposed from the radicals to the diradicals, changing the spin-polarizing operator from $K_{a}$ to $K_{a}+K_{b}$ for the triplet state and $K_{a}-K_{b}$ for the singlet. One may trace as well the interference between correlation and spin polarization and the existence of cooperative effects increasing the coefficients of the $1 \mathrm{~h}-1 \mathrm{p}$ spin polarizing contributions. For the triplet state, one may use a single reference expansion from the $m_{s}=1$ component, and a coupled cluster expansion faces the fact that a second spin-flip excitation implies some operators that do not act on the reference. Although the singlet-state treatment requires an expansion from two reference determinants, and thus two wave operators, the same problem appears for each of them.

\section{DISCUSSION: A METHODOLOGICAL CHALLENGE}

We have reported the observed and unexpected increase in coefficients of spin-polarizing $1 \mathrm{~h}-1 \mathrm{p}$ determinants when pushing the CI expansion to full valence CI. We have tried to understand the origin of the phenomenon, concerning apparently both monoradicals and di-radicals. Two mechanisms certainly enter in this behavior, namely, a positive interference between spin polarization and correlation and a cooperative aspect of the spin polarization.

Cooperative effects are fundamental in phase transitions of materials, which involve molecular reorganizations under pressure, temperature, or external field. In the present problem, the cooperativity concerns a purely electronic phenomenon. It is a positive cooperativity, as the spin polarization of a closed shell is enhanced by the spin polarization of the other electron pairs.

This may be formulated this way: starting from a closed-shell description of the core, the probability to get two local polarizations is larger than the product of the probabilities to get independent 
polarization excitations. This phenomenon questions the relevance of the standard tool for the description of electronic systems, namely, the coupled-cluster representation of the wave function, when treating spin-polarization phenomena. In this formalism, based on perturbation theory and diagrammatic representations, the amplitude of a quadruply excited determinant is obtained from the product of the amplitudes of complementary pairs of double excitations leading to this quadruply excited determinant from the reference function. ${ }^{29-32}$ This formalism is based on many-body perturbation theory $^{33}$ and is valid under two conditions, namely,

(i) when $\left|\Phi_{\alpha}\right\rangle=T_{i}^{+} T_{j}^{+}\left|\Phi_{0}\right\rangle$ is a second-generation determinant, the interactions between it and the first generation determinants are equal to the interactions between the reference and the first generation determinants, namely,

$$
\left\langle T_{i}^{+} \Phi_{0}|H| \Phi_{\alpha}\right\rangle=\left\langle\Phi_{0}|H| T_{j}^{+} \Phi_{0}\right\rangle
$$

and

$$
\left\langle T_{j}^{+} \Phi_{0}|H| \Phi_{\alpha}\right\rangle=\left\langle\Phi_{0}|H| T_{i}^{+} \Phi_{0}\right\rangle,
$$

i.e., if the interactions relative to two determinants related by an excitation operator only depend on the excitation operator, and

(ii)

$$
\begin{aligned}
\left\langle\Phi_{\alpha}|H| \Phi_{\alpha}\right\rangle-\left\langle\Phi_{0}|H| \Phi_{0}\right\rangle= & \left\langle T_{i}^{+} \Phi_{0}|H| T_{i}^{+} \Phi_{0}\right\rangle-\left\langle\Phi_{0}|H| \Phi_{0}\right\rangle \\
& +\left\langle T_{j}^{+} \Phi_{0}|H| T_{j}^{+} \Phi_{0}\right\rangle-\left\langle\Phi_{0}|H| \Phi_{0}\right\rangle
\end{aligned}
$$$$
\text { if }
$$

i.e., if excitation energies are additive.

Under these two conditions, the coefficient of $\left|\Phi_{\alpha}\right\rangle$ contains the product of the coefficients of $\left|T_{j}^{+} \Phi_{0}\right\rangle$ and $\left|T_{i}^{+} \Phi_{0}\right\rangle$. If one of these conditions is not satisfied, one must introduce specific many-body operators $T_{\alpha}^{+}\left|\Phi_{\alpha}\right\rangle=T_{\alpha}^{+}\left|\Phi_{0}\right\rangle$ in the wave operator, with their specific amplitudes, to get the exact coefficients of the multiply excited determinants. There are two ways of breaking these conditions.

The first one concerns the additivity of excitation energies, which is, in general, only approximate, but which may be strongly violated in some specific problems. Consider, for instance, a onedimensional antiferromagnetic chain of spins with its associated Néel reference determinant. Performing spin exchange between two adjacent sites, say, $k$ and $l$, induces two spin frustrations and costs $2 J$, with $J$ being the inter-site magnetic coupling. Let us call $c$ the amplitude of this operation. As excitation energies are additive, performing a second spin exchange on a remote bond costs $4 J$, and indeed, the coefficient of the corresponding determinant may be approximated by $c^{2}$. If a second spin exchange now concerns atoms $m$ and $n$, close to $l$, the excitation energy is only $2 J$, it is no longer additive, and the coupled-cluster approximation fails, or must be corrected. ${ }^{34,35}$ In this problem, spin exchange on a given bond facilitates that on neighbor bonds-this can be considered as a "denominator driven" cooperative effect.

A second deviation concerns transferability of the interactions. This transferability is satisfied when the operators are double excitations, which are the fundamental processes in the electronic correlation problem. It is no longer the case for single excitations since the interactions they generate depend on the determinant on which they act. In the correlation problem, Brillouin's theorem ensures that the corresponding amplitudes are small and the defect of introducing products of $t^{(1)}$ amplitudes is not problematic. However, one sees in our problem that the spin-polarization effects, which, in principle, concern various electron pairs, are not additive, due to a positive interference effect, which increases the intensity of the interactions of the same $1 \mathrm{~h}-1 \mathrm{p}$ operators, when going to multiple excitations. One may consider this cooperative effect as "interaction-driven." The numerical examples demonstrate that it should be necessary to consider multiple spin-polarization operators to correctly treat their elementary amplitudes.

The problem of a correct treatment of spin polarization is not a purely academic challenge: most of the chemical rearrangements proceed through barriers where the molecules take a diradical character and which are accordingly subject to collective spin polarization effects. Treating them from a single reference approach, or even from a two-configuration approach, may miss this effect and overestimate the barrier height of the reaction. Intensive CI calculations able to treat cooperative effects correctly are not possible in large systems, especially remembering that, in conjugated hydrocarbons, spin polarization also concerns the $\sigma$ frame and that cooperativity is certainly present between spin polarizations of $\sigma$ cores and $\pi$ electrons. The unrestricted self-consistent formalisms might appear as an alternative, since they actually introduce multiplicative effects due to their iterative character, but they also miss spin-flip contributions and destroy spin-symmetry requirements. An interesting treatment may start from a spin-unrestricted treatment and proceed to an appropriate symmetry-restoring projection, as done by JiménezHoyos et al., ${ }^{36-38}$ but it is likely that the resulting wave function will anyway expand on multiply excited configurations, increasing the cost of the final calculation of energy.

\section{SUPPLEMENTARY MATERIAL}

See the supplementary material for (I) optimized geometries of $\mathrm{CH}_{3}$ and $\mathrm{C}_{12} \mathrm{H}_{4}$, and (II) CI calculations: computational details, input files, and configurations for the two systems.

\section{DATA AVAILABILITY}

The data that support the findings of this study are available within the article (and its supplementary material).

\section{REFERENCES}

${ }^{1}$ H. M. McConnell, J. Chem. Phys. 24, 460 (1956).

${ }^{2}$ S. I. Weissman, J. Chem. Phys. 25, 890 (1956).

${ }^{3}$ B. Venkataraman and G. K. Fraenkel, J. Chem. Phys. 24, 737 (1956).

${ }^{4}$ R. Bersohn, J. Chem. Phys. 24, 1066 (1956).

${ }^{\mathbf{5}}$ H. Kollmar and V. Staemmler, Theor. Chim. Acta 48, 223 (1978).

${ }^{6}$ A. F. Voter, M. M. Goodgame, and W. A. Goddard, Chem. Phys. 98, 7 (1985).

${ }^{7}$ G. Berthier, J. Chim. Phys. 51, 363 (1954).

${ }^{8}$ J. A. Pople and R. K. Nesbet, J. Chem. Phys. 22, 571 (1954).

${ }^{9}$ A. P. Ginsberg, J. Am. Chem. Soc. 102, 111 (1980).

${ }^{10}$ L. Noodleman, J. Chem. Phys. 74, 5737 (1981).

${ }^{11}$ All calculations were performed at the UB3LYP/6-311G ${ }^{* * 14}$ DFT level (Gaussian 09 package ${ }^{13}$ ) for geometry optimizations (energy gradients lower than $10^{-5}$ a.u.) and with the RASSCF procedure (MOLCAS program ${ }^{12}$ ), using ANO basis sets $^{15}$ (contracted to minimal basis sets), for the CI calculations. 
${ }^{12}$ F. Aquilante, L. De Vico, N. Ferré, G. Ghigo, P. Å. Malmqvist, P. Neogrády, T. B. Pedersen, M. Pitoňák, M. Reiher, B. O. Roos, L. Serrano-Andrés, M. Urban, V. Veryazov, and R. Lindh, J. Comput. Chem. 31, 224 (2010).

${ }^{13}$ M. J. Frisch, G. W. Trucks, H. B. Schlegel, G. E. Scuseria, M. A. Robb, J. R. Cheeseman, G. Scalmani, V. Barone, G. A. Petersson, H. Nakatsuji, X. Li, M. Caricato, A. V. Marenich, J. Bloino, B. G. Janesko, R. Gomperts, B. Mennucci, H. P. Hratchian, J. V. Ortiz, A. F. Izmaylov, J. L. Sonnenberg, Williams, F. Ding, F. Lipparini, F. Egidi, J. Goings, B. Peng, A. Petrone, T. Henderson, D. Ranasinghe, V. G. Zakrzewski, J. Gao, N. Rega, G. Zheng, W. Liang, M. Hada, M. Ehara, K. Toyota, R. Fukuda, J. Hasegawa, M. Ishida, T. Nakajima, Y. Honda, O. Kitao, H. Nakai, T. Vreven, K. Throssell, J. A. Montgomery, Jr., J. E. Peralta, F. Ogliaro, M. J. Bearpark, J. J. Heyd, E. N. Brothers, K. N. Kudin, V. N. Staroverov, T. A. Keith, R. Kobayashi, J. Normand, K. Raghavachari, A. P. Rendell, J. C. Burant, S. S. Iyengar, J. Tomasi, M. Cossi, J. M. Millam, M. Klene, C. Adamo, R. Cammi, J. W. Ochterski, R. L. Martin, K. Morokuma, O. Farkas, J. B. Foresman, and D. J. Fox, Gaussian 09, Gaussian, Inc., Wallingford CT, 2013.

${ }^{14}$ R. Krishnan, J. S. Binkley, R. Seeger, and J. A. Pople, J. Chem. Phys. 72, 650 (1980).

${ }^{15}$ P.-O. Widmark, P.-Å. Malmqvist, and B. O. Roos, Theor. Chim. Acta 77, 291 (1990).

${ }^{16}$ P. De Loth, P. Cassoux, J. P. Daudey, and J. P. Malrieu, J. Am. Chem. Soc. 103, 4007 (1981)

${ }^{17}$ C. J. Calzado, J. Cabrero, J. P. Malrieu, and R. Caballol, J. Chem. Phys. 116, 2728 (2002).

${ }^{18}$ J. P. Malrieu, R. Caballol, C. J. Calzado, C. de Graaf, and N. Guihéry, Chem. Rev. 114, 429 (2014).

${ }^{19}$ L. Salem and C. Rowland, Angew. Chem., Int. Ed. Engl, 11, 92 (1972).

${ }^{20}$ T. Stuyver, B. Chen, T. Zeng, P. Geerlings, F. De Proft, and R. Hoffmann, Chem. Rev. 119, 11291 (2019).
${ }^{21}$ The RASSCF calculations were performed using ANO basis sets ${ }^{15}$ (contraction $4 \mathrm{~s} 3 \mathrm{p} 2 \mathrm{~d}$ and $2 \mathrm{~s} 1 \mathrm{p}$ for the $\mathrm{C}$ and $\mathrm{H}$ atoms, respectively) for the $\mathrm{CI}$ calculations.

${ }^{22}$ H. C. Longuet-Higgins, J. Chem. Phys. 18, 265 (1950).

${ }^{23} \mathrm{~L}$. Salem, The Molecular Orbital Theory of Conjugated Systems (Benjamin Inc., New York, 1966).

${ }^{24}$ M.-B. Lepetit, B. Oujia, J.-P. Malrieu, and D. Maynau, Phys. Rev. A 39, 3274 (1989).

${ }^{25}$ B. Oujia, M.-B. Lepetit, D. Maynau, and J.-P. Malrieu, Phys. Rev. A 39, 3289 (1989).

${ }^{26}$ A. A. Ovchinnikov, Theor. Chim. Acta 47, 297 (1978).

${ }^{27}$ J.-P. Malrieu, N. Guihéry, C. J. Calzado, and C. Angeli, J. Comput. Chem. 28, 35 (2007).

${ }^{28}$ S. Shaik and P. C. Hiberty, A Chemist's Guide to Valence Bond Theory (John Wiley \& Sons, Inc., Hoboken, NJ, USA, 2007).

${ }^{29}$ F. Coester, Nucl. Phys. 7, 421 (1958).

${ }^{30}$ F. Coester and H. Kümmel, Nucl. Phys. 17, 477 (1960).

${ }^{31}$ J. Čížek, J. Chem. Phys. 45, 4256 (1966).

${ }^{32}$ R. J. Bartlett and G. D. Purvis, Int. J. Quantum Chem. 14, 561 (1978).

${ }^{33}$ J. Goldstone, Proc. R. Soc. London, Ser. A 239, 267 (1957).

${ }^{34}$ B. Miguel and J.-P. Malrieu, Phys. Rev. B 54, 1652 (1996).

${ }^{35} \mathrm{M}$. Al Hajj and J.-P. Malrieu, Phys. Rev. B 70, 184441 (2004).

${ }^{36}$ C. A. Jiménez-Hoyos, T. M. Henderson, T. Tsuchimochi, and G. E. Scuseria, J. Chem. Phys. 136, 164109 (2012).

${ }^{37}$ P. Rivero, C. A. Jiménez-Hoyos, and G. E. Scuseria, J. Phys. Chem. A 117, 8073 (2013).

${ }^{38}$ T. Tsuchimochi and G. E. Scuseria, J. Chem. Phys. 134, 064101 (2011).

${ }^{39}$ V. Veryazov, GV, 2014.

${ }^{40}$ F. Aquilante, T. B. Pedersen, V. Veryazov, and R. Lindh, Wiley Interdiscip. Rev.: Comput. Mol. Sci. 3, 143 (2013). 